\title{
DIFFICOLTÀ DI APPRENDIMENTO DEI TEMPI E MODI VERBALI DELL'ITALIANO L2 DA PARTE DEGLI APPRENDENTI SERBOFONI
}

\author{
NEVENA CEKOVIĆ-RAKONJAC ${ }^{1}$ \\ Università di Belgrado, Facoltà di Filologia \\ Dipartimento di italianistica \\ Belgrado, Serbia
}

Kroz kontrastivnu analizu i analizu grešaka u radu se ilustruju teškoće srpskih govornika u učenju italijanskog kao drugog/stranog jezika na morfosintaksičkom nivou, prvenstveno u vezi sa oblicima i upotrebom glagolskih vremena i načina. Istovremeno, ukazuje se i na međujezički nivo na kojem se takve poteškoće najčešce manifestuju, s namerom da se nastavnicima pruže korisne smernice za osmišljavanje ciljanih didaktičkih aktivnosti koje bi uzele u obzir specifične jezičke potrebe srbofonih studenata.

Ključne reči: italijanski J2, srbofoni učenici, kontrastivna analiza, analiza grešaka, glagolski sistem.

\section{INTRODUZIONE}

Basandoci sull'Analisi contrastiva e l'Analisi degli errori (Đorđević 2000, 0dlin 2003, Gass \& Selinker 2008), ci siamo prefissati l'obiettivo di illustrare con questo contributo, in maniera più sintetica possibile, le difficoltà che un discente di madrelingua serba (L1) incontra lungo il suo percorso di apprendimento dell'italiano come lingua seconda/ straniera (L2), precisando, ove possibile, in quale fase dell'interlingua tali difficoltà solitamente appaiono. Ci siamo però limitati a discutere in questa sede soltanto la problematica relativa alle forme ed agli usi dei tempi e dei modi verbali, ${ }^{2}$ suddividendola per sezioni dedicate ai tempi verbali (ovvero all'aspetto verbale e al confronto dei relativi sistemi nelle due lingue), alla forma attiva e passiva, ai modi (in)definiti, alla concordanza

1 Kontakt podaci (Email): n.cekovic@fil.bg.ac.rs

2 Per approfondimenti riguardo alle difficoltà a livello fonologico e ortografico si veda rispettivamente: Ceković-Rakonjac (2011a) e Ceković-Rakonjac (2011b). 
dei tempi, al periodo ipotetico. ${ }^{3}$ Nella parte conclusiva del contributo, abbiamo infine cercato di riassumere le maggiori lacune dei nostri studenti, nonché di indicare le possibili implicazioni glottodidattiche della nostra analisi.

Siamo partiti dai suddetti approcci teorici per mettere in luce il transfer negativo causato dalla L1, senza tuttavia voler con ciò gettare l'ombra sul transfer positivo dovuto alla lingua madre dell'apprendente. Sebbene dunque verranno analizzati solo gli errori causati dalle interferenze con la L1, riteniamo opportuno ricordare che non tutti gli errori possono essere interpretati in ottica della L1: oltre a considerare in questa sede la lingua madre come l'unica fonte di problemi acquisizionali, siamo consapevoli che il processo acquisizionale viene influenzato in maniera potenzialmente negativa anche da altri fattori linguistici ed extralinguistici.

\section{TEMPI}

\subsection{ASPETTO VERBALE}

La categoria del tempo è strettamente legata ad un'altra categoria tipica delle lingue slave: I'aspetto dei verbi, il quale in serbo, oltre al modo e al tempo, fornisce informazioni anche sulla durata dell'azione. Data l'inesistenza dell'aspetto verbale in questo senso nella lingua italiana, si potrebbe dire che l'aspettualità di un verbo serbo si rispecchia in italiano nell'uso dei modi e dei tempi verbali.

In serbo si hanno, dunque, verbi imperfettivi o durativi („nesvršeni") e verbi perfettivi o momentanei (,svršeni glagoli"). Vengono solitamente formati dalla stessa radice e nel caso dei perfettivi, vengono abbondantemente sfruttate la suffissazione e la prefissazione, la quale a volte introduce un cambiamento di significato. "Per esempio, „piti" (bere) ha i momentanei: „popiti" (bere un po'), , ispiti" (bere tutto), „napiti se" (bere a sazietà) , ,opiti se" (ubriacarsi) ed altri" (Cronia 1959: 63). "I verbi imperfettivi esprimono l'azione nel suo compiersi, nel suo scorrere, i verbi perfettivi, invece, esprimono la realizzazione dell'azione, il processo compiuto o la durata dell'azione prima del suo compimento" (Lipovac Gatti 1997: 84). Ai fini illustrativi, presentiamo alcuni verbi italiani in cui è possibile riscontrare delle analogie con i verbi imperfettivi (citati per primi) e perfettivi serbi: „učiti” (studiare) - "naučiti” (imparare); „sedeti” (stare seduto, sedere) "sesti" (sedersi); „ležati" (giacere) - „leći" (sdraiarsi); „gledati" (guardare) - „pogledati” (dare un'occhiata); „, šetati" (passeggiare) - „prošetati" (fare una passeggiata) ; „pomagati" (aiutare) - „pomoći" (dare un aiuto) ; „gristi" (mordere) - „Zagristi” (dare un morso).

In che modo, allora, viene resa in italiano L2 l'idea dell'(im)perfettività da parte dello studente serbo? Non è un compito facile, davanti ad una vasta gamma di tempi e modi italiani, capire che in linea di massima il Presente e l'Imperfetto corrispondono all'idea dell'imperfettività, mentre invece il Passato prossimo, il Passato remoto e il Trapassato prossimo rendono l'idea della perfettività. Tale compito diventa ancora

3 Gli esempi di cui ci siamo serviti per illustrare gli errori tipici degli apprendenti sono frutto dell'esperienza dell'autore nell'insegnamento di lingua italiana presso la Facoltà di filologia di Belgrado a partire dal 1999, e sono basati sui compiti scritti e orali degli apprendenti di I, II e III anno di studi (livelli di competenza: basico, intermedio e avanzato). 
più difficile quando lo studente si rende conto che in questi casi non esiste una corrispondenza assoluta tra le due lingue a confronto: "A proposito dei tempi passati in serbo e in italiano, è sbagliato identificare automaticamente il verbo imperfettivo serbo con l'imperfetto italiano e il verbo perfettivo serbo al passato con il passato prossimo oppure il passato remoto italiani. Tali coincidenze sono solamente parziali, dato che l'uso dei tempi passati in italiano si basa su regole diverse rispetto a quelle che valgono per l'uso dei tempi passati in serbo. Confronta: "Era ImperfetTo una bella giornata („Bio je lep dan"); Ė stata PASSATo PRossimo una bella giornata („Bio je lep dan")" (Moderc 2004a: 263). È possibile, in effetti, riscontrare certi casi in cui, da un lato, i verbi imperfettivi corrispondono, oltre all'Imperfetto, anche al Passato prossimo, al Passato remoto e al Trapassato prossimo e dall'altro lato, i verbi perfettivi vengono resi anche con I'Imperfetto (oltre che con il Passato prossimo, il Passato remoto e il Trapassato prossimo) ${ }^{4}$. Sono comprensibili allora le difficoltà e i dubbi degli studenti serbofoni rispetto all'uso dei tempi passati italiani.

Il serbo, inoltre, grazie alla capacità di distinguere l'aspetto verbale imperfettivo da quello perfettivo, ha praticamente ridotto il proprio sistema di tempi passati ad uno solo: il Perfetto (corrispondente del Passato prossimo italiano), di carattere quasi universale e più frequentemente utilizzato tra tutti i tempi del passato. Il Perfetto serve quindi a esprimere sia l'idea di passato che la nozione di anteriorità rispetto al passato: di conseguenza, lo studente non solo fatica a distinguere il Passato prossimo e l'Imperfetto italiani, ma gli risulta molto difficile anche il tentativo di rendere l'anteriorità per mezzo del Trapassato prossimo italiano. A causa di tutto ciò, è possibile nella prassi didattica riscontrare errori come: * Tutto il giorno ieri ascoltavo la musica (,Juče sam ceo dan slušao muziku" ",Islušati" - imperfettivo); *Sono state le cinque quando mi ha chiamato („Bilo je pet sati kad me je pozvao"); *Non ho voluto vedere quel film perché l'ho già visto due mesi prima („Nisam hteo da gledam taj film jer sam ga već gledao pre dva meseca”).

\subsection{TEMPI VERBALI A CONFRONTO}

Il serbo dispone di un sistema composto di 7 tempi verbali che vediamo ora a confronto con quelli italiani:

\begin{tabular}{|c|c|}
\hline ITALIANO & SERBO \\
\hline Presente & Presente $\left({ }_{\text {„P}}\right.$ Prezent $\left.{ }^{\prime \prime}\right)$ \\
\hline Passato prossimo & Perfetto („Perfekat") \\
\hline Imperfetto & Imperfetto („Imperfekat") \\
\hline Passato remoto & Aoristo (,Aorist') \\
\hline Trapassato prossimo/remoto & Piuccheperfetto (,Pluskvamperfekat) \\
\hline Futuro & Futuro (,Futur I') \\
\hline Futuro anteriore & Futuro anteriore (,Futur II') \\
\hline
\end{tabular}

Tabella 1: Tempi verbali a confronto

4 Per approfondimenti v. Moderc (1995) e Moderc (2004b). 


\subsubsection{PRESENTE}

Riguardo all'uso del Presente italiano, in genere non si rivelano particolari difficoltà negli apprendenti serbofoni, se non in certi casi in cui lo studente cerca di riprodurre la forma dell'infinito anche all'interno del paradigma verbale dimenticando che si tratta di forme atipiche e/o irregolari: *io piangio (dal verbo piangere); *io spedo (dal v. spedire); *io uscio (dal v. uscire).

\subsubsection{PASSATO PROSSIMO}

Oltre a quanto già accennato a proposito dell'aspetto verbale, ci pare opportuno sottolineare che per analogia con il serbo, dove il Perfetto domina gli altri tempi passati, gli studenti tendono a fossilizzarsi sul sovvrauso del Passato prossimo italiano, non sfruttando la vasta gamma dei tempi passati che la lingua italiana gli mette a disposizione.

Relativamente invece alla formazione del Passato prossimo (così come degli altri tempi passati e di tutti i tempi composti in generale) la difficoltà più spiccata nell'ottica del discente riguarda la scelta dell'ausiliare e l'accordo del participio. Per formare il Perfetto in serbo viene usato sempre lo stesso ausiliare (,, biti" $^{\prime \prime}$ - essere) e il participio accorda sempre al numero e al genere con il soggetto. Sono comprensibili allora gli errori come: *Lei ha detta (,ona je rekla"), *Noi siamo viaggiati („, mi smo putovali"), soprattutto ai livelli basici.

\subsubsection{IMPERFETTO}

L'Imperfetto serbo, data l'esistenza dell'aspetto verbale, si usa molto di meno che in italiano e viene quasi sempre sostituito dal Perfetto dei verbi imperfettivi (il Perfetto serve a sostituire anche I'Aoristo serbo (v. 2.2.4), visto che sia l'Aoristo che l'Imperfetto vengono percepiti come forme arcaiche). ${ }^{5}$

\subsubsection{PASSATO REMOTO}

La corrispondenza tra il Passato remoto italiano e il suo equivalente serbo è parziale, dato che l'Aoristo serbo, oltre ovviamente a poter riferirsi agli eventi remoti, può far riferimento anche ad un passato immediato, dove coincide con l'uso del Passato prossimo italiano. Nonostante quindi sia vivo nel parlato, l'Aoristo è una forma verbale in genere non molto usata: nella lingua serba contemporanea infatti, viene usato perlopiù per esprimere un'azione compiuta nel passato recente, mentre per esprimere le azioni compiute in un passato piuttosto remoto gli viene preferito il Perfetto dei verbi perfettivi, essendo l'Aoristo percepito come arcaico. Inoltre, "la forma serbocroata sottolinea l'azione compiuta senza precisare il periodo temporale in cui questa viene eseguita (passato, presente, futuro), e indica un particolare interesse del parlante per le vicende che sta esponendo, riattualizzandole; il passato remoto è invece un puro preterito, e perciò non può riferirsi né al presente né al futuro, e come tale si limita a una semplice constatazione verso la quale il parlante non nutre alcun interesse

5 Sugli errori tipici legati all'uso dell'Imperfetto italiano si veda la sezione dedicata all'Aspetto verbale. 
soggettivo" (Savić 1980: 75-76). È comprensibile allora che, partendo dalla propria L1, lo studente serbo faccia fatica ad acquisire gli usi del Passato remoto italiano. Riteniamo inoltre altrettanto importante mettere in evidenza la difficoltà che lo studente riscontra nell'acquisizione delle complesse ed arcaiche forme del paradigma verbale in questione.

\subsubsection{TRAPASSATO PROSSIMO E TRAPASSATO REMOTO}

Tra tutti i tempi verbali del passato, l'uso del Trapassato prossimo rappresenta indubbiamente una delle maggiori difficoltà per un apprendente serbo. Il suo corrispondente serbo viene utilizzato molto di meno (a favore del Perfetto). Occorre quindi sensibilizzare lo studente all'anteriorità espressa dal Trapassato prossimo italiano allo scopo di evitare errori come: *Ero stanco perché il giorno prima ho lavorato tanto ("Bio sam umoran jer sam prethodni dan puno radio"). Lo stesso discorso vale anche per il Trapassato remoto, il quale tra I'altro non ha una forma verbale corrispondente in serbo. ${ }^{6}$

\subsubsection{FUTURO SEMPLICE E FUTURO ANTERIORE}

Relativamente all'uso dei due tempi italiani non si rivelano grosse difficoltà nei discenti se non quando si tratta del loro valore modale. Sebbene anche il Futuro serbo (insieme ad altri mezzi) ${ }^{7}$ possa veicolare la modalità ed indicare quindi un dubbio 0 una supposizione, la stessa funzione modale in italiano $\mathrm{L} 2$ risulta piuttosto difficile da apprendere spesso anche fino ai livelli avanzati di interlingua. Per questi motivi, gli studenti diranno: Dov'è Mauro? piuttosto che: Dove sarà Mauro? („Gde (li) je Mauro?"); Dov'è andato? invece di Dove sarà andato? (",Gde (li) je otišao?"). Riflettendo inoltre sull'uso del Futuro anteriore serbo nelle subordinate temporali e condizionali (e premettendo che non si tratta di un vero tempo anteriore dato che esprime azioni contemporanee ad altre azioni in futuro, per cui può essere usato solo nelle subordinate, a differenza dell'italiano), ${ }_{1}^{8} \mathrm{ci}$ sono da aspettarsi degli errori di transfer come: *Quando avrai avuto tempo, chiamami! (, Kad budeš imao vremena, pozovi me!"); *Se sarai venuto, chiamami! („Ako budeš dolazio, javi mi se!”).

\section{FORMA ATTIVA E PASSIVA}

A differenza dell'italiano, che usufruisce abbondantemente della forma passiva, il serbo, pur avendo a disposizione entrambe le forme, predilige utilizzare quella attiva. Di conseguenza, gli apprendenti serbi tendono ad usare maggiormente la forma attiva anche in italiano L2 ed in genere soltanto ai livelli di interlingua avanzata si può aspettare un uso adeguato della forma passiva (anche nel parlato).

6 Va accennato che il Trapassato remoto sta quasi completamente scomparendo dall'italiano contemporaneo, soprattutto quello parlato e quello usato dai mass media, rimanendo tuttavia presente nelle opere letterarie dove a sua volta si sottopone alle regole sintattiche d'uso piuttosto severi.

7 Quali, ad esempio: i costrutti col verbo essere al futuro "biće da"; I'espressione „može biti da" (può darsi che); gli avverbi di giudizio "možda" (forse) , ,I(vrlo) verovatno" ((molto) probabilmente); I'uso del Presente, nel caso del Futuro semplice, o del Passato, nel caso del Futuro anteriore.

8 Si pensi agli esempi come: Quanto tu tornerai, io sarò già partito (si ringrazia per la gentile osservazione il prof. Ivan Klajn). 


\section{MODI}

\subsection{MODI FINITI: CONGIUNTIV0, CONDIZIONALE E IMPERATIV0}

Una serie di errori che l'apprendente serbo può commettere riguardo all'uso del Congiuntivo risultano indubbiamente dall'inesistenza di tale forma in serbo. Sebbene ai livelli di varietà avanzate gli studenti riescano a padroneggiare in maniera soddisfacente questa forma, alcuni dei suoi usi particolari rimangono pur sempre difficili da acquisire, così come negli esempi: *Non è che è stanco, ha solo dormito poco („Nije da je umoran, samo je nedovoljno spavao"), *Cerco un uomo che saprebbe ascoltarmi ( „Tražim muškarca koji bi umeo da me sasluša").

A proposito del Condizionale italiano, il suo corrispondente serbo, il Condizionale ("Kondicional") può essere fonte di certi errori di transfer, dovuti in particolare al suo valore iterativo che serve per indicare un'azione che si è ripetuta nel passato e che, tra l'altro viene reso in italiano con l'Imperfetto, ad es: *Quando ero al mare... andrei ogni giorno in spiaggia e prenderei il sole (, Kad sam bio na moru...odlazio bih svaki dan na plažu i sunčao bih se"). Inoltre, risulta particolarmente difficile per uno studente serbo impadronirsi dell'uso del condizionale composto, dato che la corrispondente forma della sua L1 („,Kondicional prošli") è quasi scomparsa dall'uso quotidiano. Invece di dire, per es.: Chi l'avrebbe pensato! lo studente serbo preferisce in effetti esprimersi nel seguente modo: *Chi lo penserebbe! *Chi I'ha pensato! Chi lo poteva pensare! („„Ko je to mogao da pomisli?"). ${ }^{9}$

L'imperativo italiano ha come corrispondente in serbo l'Imperativo (,Imperativ") e di solito non crea tanti problemi agli apprendenti, se non nella sua forma negativa alla $2^{\text {a }}$ persona singolare. Malgrado anche in serbo esista una formula espressiva contenente I'infinito, ai fini di una maggiore enfasi viene solitamente preferita la negazione non seguita da un'apposita forma derivante dal tema del presente. Ne risultano errori come: *Non scrivi! („Ne piši!" cfr. „,Nemoj pisati!"), *Non guardi! (,Ne gledaj!" cfr. „,Nemoj gledati!"), ecc.

\subsection{MODI INDEFINITI}

Ecco a confronto i modi indefiniti nelle due lingue:

\begin{tabular}{|l|l|}
\hline \multicolumn{1}{|c|}{ ITALIANO } & \multicolumn{1}{c|}{ SERBO } \\
\hline Infinito presente & „Infinitiv" \\
\hline Infinito passato & - \\
\hline Participio presente & - \\
\hline Participio passato & "Glagolski pridev radni" \\
& "Glagolski pridev trpni" \\
\hline Gerundio presente & "Glagolski prilog sadašnji" \\
\hline Gerundio passato & "Glagolski prilog prošli" \\
\hline
\end{tabular}

Tabella 2: Modi indefiniti a confronto

9 Per approfondimenti sull'analisi contrastiva del condizionale v. Tekavčić (1982: 7-8). 


\subsubsection{INFINITO}

Premesso che la lingua serba in una serie di proposizioni subordinate preferisce in genere la forma esplicita, anche se le strutture implicite non le sono del tutto estranee, è naturale aspettarsi che questa differenza tra le due lingue crei una serie di difficoltà all'apprendente. Sebbene a volte usare l'infinito significhi per lui prendere una via più semplice (per evitare, ad es. le "trappole" del congiuntivo) e stilisticamente più plausibile, lo studente di solito fatica ad acquisirlo e tende a preferire le forme esplicite fino ai livelli intermedi. Invece di: Penso di aver lasciato le chiavi in macchina, dice piuttosto *Penso che ho lasciato le chiavi in macchina ("Mislim da sam ostavio ključeve u kolima"), anche perché, come si è visto dallo schema, non esiste una forma corrispondente all'infinito passato nella sua $\mathrm{L} 1$.

La differenza tra le due lingue si rispecchia anche nell'uso delle preposizioni abbinate all'infinito. Mentre in italiano l'infinito viene spesso preceduto dalla preposizione, tale fenomeno in serbo è piuttosto raro ed è frutto di influssi esogeni (cfr. C'è qualcosa da mangiare? „Ima li nešto za pojesti? Ima li nešto da se jede?"). Per tale motivo, soprattutto nelle varietà iniziali, è possibile riscontrare esempi come: *Vado vedere, *Corro per/a prendo I'autobus, *Lui comincia di ridere, *Desidero di andare.

Osserviamo più dettagliatamente alcuni errori legati all'uso dell'infinito nelle proposizioni oggettive e soggettive:

- $\quad$ con i verbi ed espressioni che indicano desiderio o timore: con i verbi modali la forma più diffusa in serbo è composta di „ $d a^{\prime \prime}(c h e)+$ presente. Ne risultano spesso, ai livelli prebasici soprattutto, strutture del tipo: *voglio che io vado („želim da idem"), *devo che mangio („moram da jedem”). In questi casi è possibile utilizzare anche l'infinito in serbo, solo che tale struttura risulta molto meno diffusa e più tipica del croato, eppure produce errori di confusione come: *devo che partire („moram poći/da pođem”);

- $\quad$ con i verbi di significato dichiarativo: anche con verba dicendi lo studente preferisce utilizzare la forma esplicita, creando così frasi del tipo: *ti dico che vai („,kažem ti da ideš"), *ti chiedo che mi compri („,molim te da mi kupiš");

- $\quad$ con i verbi che esprimono giudizio: si usa preferibilmente la forma esplicita, trascurando inoltre il congiuntivo, data la sua assenza in serbo. Per es.: *penso che ho ragione (",mislim da sam u pravu");

- $\quad$ con i verbi che indicano un sentimento: similmente ai casi precedenti, anche con verba sentiendi si hanno produzioni come: *sono arrabbiato che tu non mi hai detto niente („ljut sam što mi nisi ništa rekao");

- $\quad$ con i verbi impersonali: per gli stessi motivi, si incontrano casi come: *mi sembra che ho capito ("čini mi se da sam razumeo");

- $\quad$ nei cosiddetti costrutti con accusativo + infinito dopo verba sentiendi: considerato che questi costrutti vengono resi in serbo con una forma esplicita introdotta dalla congiunzione „kako” (come), gli studenti dicono in italiano L2: *Vedo un uomo come corre (senza volerne esprimere il valore modale). 
Per quanto riguarda la forma sostantivata dell'infinito, anch'essa può diventare oggetto di molti dubbi dello studente giunto ormai a livelli avanzati di apprendimento. La sua corrispondente serba, il sostantivo verbale (sostantivo formato sulla base verbale tramite la suffissazione, per es. „čitanje"- il leggere, la lettura), induce gli apprendenti ad utilizzare l'infinito italiano anche in quei casi dove è stilisticamente sconsigliato e dove invece, in serbo, risulta del tutto regolare. Ad es. "I soldi per il viaggiare non ci sono invece del più plausibile I soldi per il viaggio non ci sono (cfr. "Novca za putovanje nema")" (Moderc 2004a: 75).

\subsubsection{PARTICIPIO}

Avendo già accennato gli errori legati al participio (si veda la sezione 2.2.2), ci limitiamo a questo punto a precisare ancora che il participio serbo, a differenza di quello italiano, accorda al numero e al genere con il soggetto della frase anche in quei casi dove, all'interno di quella stessa frase, vengono utilizzati i pronomi in funzione di complemento oggetto. Risultano dunque prevedibili le forme scorrette come: $* L i$ ho comprato io ("Ja sam ih kupio"), *Le abbiamo visto ("Videli smo ih").

\subsubsection{GERUNDIO}

L'acquisizione di questa forma di solito non crea grosse difficoltà. Si può però verificare qualche problema con la scelta dell'ausiliare nel caso del gerundio passato oppure nell'uso della perifrasi con il verbo stare + gerundio, data l'inesistenza di una tale struttura in serbo (è in effetti il compito dei verbi imperfettivi esprimere la durata di un'azione). Ecco perché gli studenti preferiscono dire: Cosa fai in questo momento?Scrivo invece di: Cosa stai facendo? - Sto scrivendo (cfr. „Šta radiš? - Pišem”).

\section{CONCORDANZA DEI TEMPI}

La consecutio temporum rappresenta per lo studente serbo uno dei problemi più grossi e difficili da risolvere. La sua L1 non ha concordanza dei tempi o meglio dire, le proposizioni subordinate nel discorso indiretto mantengono in serbo lo stesso tempo verbale usato nel discorso diretto. Sono perciò prevedibili una serie di errori riguardo innanzitutto la concordanza al passato e non solo, dato che tali errori possono implicarne tanti altri ancora come, ad esempio, quelli legati all'uso del congiuntivo.

Per esprimere quindi la contemporaneità nel passato, nella subordinata in serbo viene usato il Presente. Ne risultano produzioni erronee tipo: *Pensavo che ha i soldi (cfr. "Mislio sam da ima para"). Nel caso della anteriorità, in serbo si usa il semplice Perfetto dal quale si avrà il Passato prossimo nelle frasi agrammaticali come questa: * Pensavo che il giorno prima ha avuto i soldi ("Mislio sam da je prethodnog dana dobio pare"). Per esprimere invece un'azione posteriore lo studente cerca di usare il semplice Futuro, proprio come in serbo: *Pensavo che il giorno dopo avrà i soldi ( „,Mislio sam da će sutradan dobiti pare"). 


\section{PERIODO IPOTETICO}

Data la differenza tra le strutture delle due lingue, anche il periodo ipotetico rappresenta una grossa difficoltà che lo studente serbo è tenuto a superare in italiano L2.

Per quanto riguarda il periodo ipotetico della possibilità, vengono utilizzati nella protasi in serbo la congiunzione " $k a d^{\prime \prime}$ (se) con il condizionale o la congiunzione " $d a^{\prime \prime}$ (se) con l'indicativo Presente. È questa la causa dell'errore più tipico degli apprendenti serbi (ma anche di molti italiani) che si riflette nell'uso del condizionale dopo la congiunzione se laddove l'italiano di norma prevede il congiuntivo Imperfetto. Per es.: *Se avrei $i$ soldi, comprerei la macchina. ("Kad bih imao para, kupio bih kola"). Oppure, a causa del presente nella protasi serba: *Se lo so, te lo direi. („,Da znam, rekao bih $\left.t i^{\prime \prime}\right)$.

Relativamente invece al periodo ipotetico della irrealtà, nella protasi serba si hanno la congiunzione "da" (se) e il Perfetto, e nell'apodosi il condizionale presente. Considerata la differenza rispetto all'italiano, si possono rilevare notevoli errori sia nella protasi che nell'apodosi. Per es.: Se lo sapevo, te l'avrei detto / *Se l'avessi saputo, te lo direi / *Se lo sapevo, te lo direi (cfr. „Da sam znao, rekao bih ti”).

\section{CONCLUSIONI}

In base a quanto esposto in questa sede, le maggiori difficoltà di apprendimento dell'italiano L2 da parte dello studente serbo riguardo alle forme e all'uso dei tempi e dei modi verbali italiani, risultano: la distinzione e il conseguente uso del Passato prossimo e dell'Imperfetto; la nozione di anteriorità espressa per mezzo del Trapassato prossimo; la possibilità di sfruttare la vasta gamma dei tempi passati italiani senza ridurli ad uno solo (il Passato prossimo, ad es.); la preferenza verso la forma attiva laddove l'italiano preferirebbe quella passiva; la necessità di una maggiore sensibilizzazione alle funzioni del congiuntivo; l'uso maggiore delle forme esplicite a sfavore di quelle implicite; la concordanza dei tempi, specialmente al passato; I'uso corretto dei tempi verbali all'interno del periodo ipotetico. Premesso che il corretto utilizzo della vasta gamma dei tempi e dei modi verbali diventa oggetto di uno studio approfondito ai livelli postbasici di acquisizione, possiamo concludere che le difficoltà succitate si incontrano generalmente ai livelli medio-avanzati di interlingua.

Seppur coscienti che la L1 rappresenti una fonte preziosa del transfer positivo, con la nostra analisi contrastiva abbiamo voluto, oltre che mettere in confronto il serbo come L1 e l'italiano come L2 rilevando soprattutto le differenze tra le due lingue, individuare in maniera più esauriente possibile gli errori tipici degli apprendenti serbofoni. La "prevedibilità" degli errori, come risultato della nostra analisi, ci aiuta a prevenirli ed a superare in modo opportuno le difficoltà che appaiono nel processo acquisizionale.

Lo scopo che ci siamo prefissati è di offrire ai docenti di italiano, sia nel contesto di insegnamento di L2 che di LS, oltre che ai ricercatori ed agli autori di materiali didattici 
(libri di testo, grammatiche contrastive, dizionari per gli apprendenti, ecc) ${ }^{10}$, uno strumento potenzialmente utile per la pianificazione di un intervento didattico mirato. Tale intervento dovrebbe presupporre la creazione di sillabi e di attività didattiche, nonché la pianificazione dei contenuti didattici, incentrando il tutto sui problemi contrastivi rilevati dalla nostra analisi e tenendo conto dei diversi stadi di acquisizione così come delle caratteristiche formali della L1 degli apprendenti.

\section{BIBLIOGRAFIA}

Ceković-Rakonjac, N. 2011a. Difficoltà di apprendimento dell'italiano L2 da parte dello studente serbo: livello fonetico. Filološki pregled 38(1), 139-149.

Ceković-Rakonjac, N. 2011b. Difficoltà di apprendimento dell'italiano L2 da parte dello studente serbo: livello ortografico. Komunikacija i kultura online 2 (2), 103-114.

Cronia, A. 1959. Grammatica della lingua serbo-croata. Milano: L. Trevisini editore.

Đorđević, R. 2000. Uvod u kontrastiranje jezika. Beograd: Filološki fakultet.

Gass, S. M. \& L. Selinker. 2008. Second language acquisition: an introductory course. New York: Routledge, 89-120.

Jernej, J. 2002. Strutture infinite. Strani jezici 32 (3-4), 131-136.

Katerinov, K. 1975. L'analisi contrastiva e l'analisi degli errori di lingua applicate all'insegnamento dell'italiano a stranieri. Rassegna Italiana di Linguistica Applicata VII (2-3), 17-69.

Lipovac Gatti, M. 1997. Grammatica della lingua croata. Milano: Hefti edizioni.

Moderc, S. 1995. Prevođenje srpskih prošlih vremena na italijanski. Neobjavljen magistarski rad, Filološki fakultet, Beograd.

Moderc, S. 2004a. Gramatika italijanskog jezika. Morfologija sa elementima sintakse. Beograd: Udruženje nastavnika italijanskog jezika Srbije.

Moderc, S. 2004b. Due sistemi a confronto: Il perfekat serbocroato ed alcuni dei suoi equivalenti italiani. Italica Belgradensia 5-6, 1-31.

Odlin, T. 2003. Cross-Linguistic Influence. In C. J. Doughty \& M. H. Long (eds.) The Handbook of Second Language Acquisition. 0xford: Blackwell Publishing, 436-486.

Savić, M. 1980. Le principali funzioni dell'aoristo serbocroato e del passato remoto italiano. Studije iz kontrastivne analize italijanskog i srpskohrvatskog jezika 1, 62-82.

Tekavčić, P. 1982. Intorno ad alcuni "ribelli" nella tipologia. Osservazioni contrastive su materiale italiano, serbocroato e latino. Studije iz kontrastivne analize italijanskog i srpskohrvatskog jezika 2, 5-22.

10 Secondo quanto ci risulta è disponibile una sola grammatica contemporanea che tratta i problemi contrastivi dei serbofoni, v. Moderc (2004a), e sono del tutto assenti appositi dizionari per gli apprendenti. Il libri di testo utilizzati nell'insegnamento di italiano LS nel contesto accademico non sono rivolti all'apprendente serbo di specifico bensì agli stranieri in generale, di varie provenienze, e di conseguenza non prendono in considerazione la problematica da noi trattata. 


\section{RIASSUNTO}

\section{DIFFICOLTÀ DI APPRENDIMENTO DEI TEMPI E MODI VERBALI DELL'ITALIANO L2 DA PARTE DEGLI APPRENDENTI SERBOFONI}

Il presente contributo mira ad illustrare le difficoltà degli apprendenti serbofoni nell'acquisizione dell'italiano L2, basandosi sui presupposti dell'Analisi contrastiva e dell'Analisi degli errori e con particolare riguardo alle forme ed agli usi dei tempi e dei modi verbali.Vengono inoltre indicati i livellidiinterlingua dove tali difficoltà solitamente si manifestano con l'intenzione di offrire ai docenti di italiano uno strumento pratico per la pianificazione didattica avente come scopo principale l'interesse linguistico dello studente serbo.

PAROLE CHIAVE: italiano L2, apprendenti serbofoni, analisi contrastiva, analisi degli errori, sistema verbale.

(Original scientific paper received 31.01.2012; revised 01.11.2012; accepted 05.11.2012) 